\title{
Simultaneous monitoring of crystalline active materials and resistance evolution in lithium-sulfur batteries
}

\author{
Yu-Chuan Chien ${ }^{a}$, Ashok S. Menon ${ }^{a}$, William R. Brant ${ }^{a}$, Daniel Brandella, Matthew J. \\ Lacey ${ }^{\mathrm{a}, \mathrm{b}, *}$ \\ a Department of Chemistry - Ångström Laboratory, Uppsala University, Box 538, Lägerhyddsvägen \\ 1, 75121 Uppsala, Sweden \\ b Scania CV AB, 15187 Södertälje, Sweden
}

\begin{abstract}
Operando X-ray diffraction (XRD) is a valuable tool for studying secondary battery materials as it allows for the direct correlation of electrochemical behavior with structural changes of crystalline active materials. This is especially true for the lithium-sulfur chemistry, in which energy storage capability depends on the complex growth and dissolution kinetics of lithium sulfide $\left(\mathrm{Li}_{2} \mathrm{~S}\right)$ and sulfur $\left(\mathrm{S}_{8}\right)$ during discharge and charge, respectively. In this work, we present a novel development of this method through combining operando XRD with simultaneous and continuous resistance measurement using an Intermittent Current Interruption (ICI) method. We show that a coefficient of diffusion resistance, which reflects the transport properties in the sulfur/carbon composite electrode, can be determined from analysis of each current interruption. Its relationship to the established Warburg impedance model is validated theoretically and experimentally. We also demonstrate for an optimized electrode formulation and cell construction that the diffusion resistance increases sharply at the discharge end point, which is consistent with the blocking of pores in the carbon host matrix. The combination of XRD with ICI allows for a direct correlation of structural changes with not only electrochemical properties but also energy loss processes at a non-equilibrium state, and therefore is a valuable technique for the study of a wide range of energy storage chemistries.
\end{abstract}

\section{Introduction}

Lithium-sulfur (Li-S) batteries have been attracting much research attention due to the high theoretical specific energy, $2552 \mathrm{Wh} \mathrm{kg}^{-1}$, and the abundance of sulfur. ${ }^{2}$ The system consists of a metallic lithium negative electrode and a positive electrode, most typically made from a mixture of elemental sulfur and micro-/mesoporous carbon matrix, which facilitates the electrochemical reactions of the insulating sulfur species. ${ }^{1}$ The theoretical specific energy is calculated by assuming complete sulfur utilization, which provides a theoretical capacity of $1672 \mathrm{mAh} \mathrm{g}^{-1} .^{1}$ However, only around $1000 \mathrm{mAh} \mathrm{g}^{-1}$ reversible capacity is demonstrated in most academic studies to date. ${ }^{3-5}$ The complicated reaction mechanism in the positive electrode is one of the reasons for the low utilization of active material. ${ }^{6}$ Upon discharging, elemental sulfur goes through a series of intermediate reactions and is eventually reduced to lithium sulfide $\left(\mathrm{Li}_{2} \mathrm{~S}\right)$. The intermediate products $\left(\mathrm{Li}_{2} \mathrm{~S}_{\mathrm{x}}, \mathrm{x}=2-8\right.$, also known as polysulfides) are soluble to various extents in common ether-based electrolytes ${ }^{1}$. In other words, the active material of the positive electrode dissolves and precipitates in each course of discharge or charge. The growth of the insulating sulfur and $\mathrm{Li}_{2} \mathrm{~S}$ crystals can deteriorate the conductive porous carbon matrix of the electrode by blocking pores and covering electronically conductive surface area, resulting in incomplete reaction and reduced capacity. ${ }^{6}$ Therefore, it is crucial to investigate the dissolution and precipitation of the insulating solid species and their impact on the pore structure of the positive electrode during the operation of this electrochemical system.

$X$-ray diffraction (XRD) has been demonstrated to be a powerful technique to follow the evolution 
of crystalline sulfur and $\mathrm{Li}_{2} \mathrm{~S}$ as the electrochemical reactions take place. ${ }^{7-16}$ The possibility to perform operando measurements is advantageous for the investigation of the Li-S system since ex situ experiments cannot capture the fast kinetics of interconversion between the sulfur species during a changing equilibrium. ${ }^{6}$ Nevertheless, there are several experimental challenges to overcome for operando experiments. In order to obtain a good signal-to-noise ratio from the XRD measurement of operating batteries, custom operando electrochemical cells are required. Unfortunately, the modifications in these custom cells often lead to significant deviations in the electrochemical behavior from that of a conventional cell format. First, the amount of electrolyte is often unreported in literature or too high compared to a cell optimized for specific energy. ${ }^{17}$ The electrolyte-to-sulfur (E/S) ratio has at the same time been demonstrated to be influential to many electrochemical features, such as internal resistance, Coulombic efficiency (CE) and cycle life. ${ }^{18,19}$ Another important issue is the uniformity of both the stack pressure and the electrical conductivity of a modified cell for operando XRD. It has, for example, been shown experimentally and computationally that the electrode reactivity is affected by both of these factors in a $\mathrm{LiFePO}_{4}-\mathrm{Li}$ cell. ${ }^{20}$ Aiming to tackle the above-mentioned issues, a modified coin cell targeting operando XRD measurements is designed and presented in this work.

Electrochemical methods are useful tools to characterize the porous carbon electrodes, ${ }^{21}$ especially electrochemical impedance spectroscopy (EIS). ${ }^{22}$ Since the capacitive behavior of porous carbon electrodes can be described by the transmission-line model, ${ }^{23}$ the Warburg element, which results from the analytical treatment of the model, represents a porous carbon matrix filled with electrolyte in an equivalent circuit model (ECM) and depicts its transport properties. ${ }^{22}$ Additionally, EIS also provides information on the electrolyte and charge transfer resistances, ${ }^{24,25}$ which are also valuable for studying an electrochemical system. Despite the desired functionality of EIS, it is difficult to perform EIS measurements frequently and continuously for a long period since fitting the spectra to an ECM is often desired, which necessitates manual processing and complex analysis of a large amount of data. Moreover, the system is generally required to stay invariant during the measurement. This often requires relaxing the system under open-circuit conditions to reach a steady state, which in turn interrupts the cycling and violates the principle of an operando experiment. ${ }^{26}$
During the XRD measurement in this work, the cell resistance was continuously measured by the Intermittent Current Interruption (ICI). The $\mathrm{ICl}$ method is a facile and non-disruptive technique, in which current pauses of up to a few seconds are inserted into galvanostatic cycling program at regular short intervals. From the potential response to each current interruption, the cell resistance can be derived. ${ }^{18}$ Due to the simplicity of the procedure, sequential resistance analyses throughout the whole range of states of charge (SoC) can be easily automated. ${ }^{27}$ It has been established that the time-independent part of the resistance can be extracted from the cycling data with an $\mathrm{ICl}$ protocol. ${ }^{18}$ Since it represents the sum of electronic, ionic and charge transfer resistances, we define it here as the internal resistance of the battery. In this work, it will be demonstrated that an extension of the $\mathrm{ICl}$ method enables the determination of the time-dependent part of the cell resistance, which corresponds to the Warburg impedance used to model diffusion processes in equivalent circuit fitting of EIS data. A coefficient proportional to the coefficient of the Warburg element can be obtained by the $\mathrm{ICl}$ method, which is here termed the coefficient of diffusion resistance. As mentioned above, it is established that the porous electrodes can be characterized as a Warburg element in an ECM, since the impedance response of a porous electrode can be considered as a result of ambipolar diffusion of ions and electrons. ${ }^{23}$ Therefore, the simultaneous application of $\mathrm{ICl}$ and operando XRD presents an opportunity to directly correlate information of mass transport characteristics and other energy loss processes to structural information in the same experiment.

This study investigates the impact of the precipitation and dissolution of the solid sulfur species on both the internal and diffusion resistances. With the transport properties of the positive electrode revealed by the diffusion resistance coefficient, the evolution of the insulating solid sulfur species is directly correlated to its impact on the functionality of the conductive porous carbon matrix. With this set of characterization tools, this work aims to develop an understanding of the limitations on sulfur utilization in an optimized positive electrode formulation for Li-S batteries.

\section{Experimental}

\subsection{Materials}

Elemental sulfur powder ( $S$, Sigma-Aldrich), Ketjenblack (KB, EC-600JD, Akzo Nobel), Super C65 (Imerys), carbon nanofibers (CNF, 20-200 $\mathrm{nm} \times 100 \mu \mathrm{m}$, Sigma-Aldrich), poly(ethylene oxide) (PEO, $M_{w} \sim 4,000,000$, Sigma-Aldrich), poly(vinylpyrrolidone) (PVP, $M_{w} \sim 360,000$, 
Sigma-Aldrich), carbon-coated Al-foil (20 $\mu \mathrm{m}$ thick, SDX, Showa Denko), beryllium discs (99+ $\%, \varnothing 16 \mathrm{~mm}, 0.25 \mathrm{~mm}$ thick, American Elements), polyimide tape $(60 \mu \mathrm{m}$ thick, Kapton $^{\circledR}$, RS), polyethylene film (PE film, Food Sealer Roll, OBH Nordica) and pouch bag (Z06, Skultuna Flexible) were used as received. Lithium metal foil (Li, Cyprus Foote Mineral, 125 $\mu \mathrm{m}$ thick) was also used as received but stored under Ar atmosphere. Lithium bis(trifluoromethanesulfonyl)imide (LiTFSI, BASF) and lithium nitrate ( $\mathrm{LiNO}_{3}$, Sigma-Aldrich) were dried at $120^{\circ} \mathrm{C}$ under vacuum overnight. 1,2-dimethoxyethane (DME, BASF) and 1,3dioxolane (DOL, Sigma-Aldrich) were dried with $3 \AA$ molecular sieves overnight. Celgard $\circledast 2400$ separators were dried under vacuum at $80^{\circ} \mathrm{C}$ overnight.

\subsection{S/C composite electrode}

The positive electrode was produced by following a previously optimized recipe ${ }^{28}$, with a composition of $65 \% \mathrm{~S}, 21 \% \mathrm{~KB}, 3.5 \% \mathrm{C} 65,3.5 \%$ CNF, $5.6 \%$ PEO, $1.4 \%$ PVP (by weight). First, S was mixed with $K B$ in a mortar and heated to $155{ }^{\circ} \mathrm{C}$ for 20 minutes. Together with the other components, the S-impregnated $\mathrm{KB}$ was weighed as dry powder and mixed with 5.5 vol $\%$ ethanol solution in deionized water. After two hours of planetary ball-milling, the slurry was cast onto a C-coated Al foil then dried at ambient conditions. The electrodes were cut into $\varnothing 13 \mathrm{~mm}$ discs and dried under vacuum at $55{ }^{\circ} \mathrm{C}$ overnight. The electrode used in the operando XRD measurement had a S-loading of $4.37 \mathrm{mgs} \mathrm{cm}^{-2}$ ( $\mathrm{g}_{\mathrm{s}}$ denotes grams of sulfur). Two electrodes with S-loadings of 4.09 and $3.83 \mathrm{mg}_{\mathrm{s}}$ $\mathrm{cm}^{-2}$ were tested in conventional CR2025format coin cells (Hohsen Corp.) with 6 and 10 $\mu \mathrm{L} \mathrm{mgs}{ }^{-1}$ electrolyte, respectively.

\subsection{Modified coin cell and Operando XRD}

A schematic diagram of our modified coin cell is presented in Figure 1 . Holes of $7 \mathrm{~mm}$ diameter were drilled in both the cap and can of the stainless steel casing of a CR2025 coin cell. The holes were sealed by Kapton ${ }^{\circledR}$ tape on both sides. The positive electrode, Celgard 2400 separator $(\varnothing 17 \mathrm{~mm})$ and the Li metal negative electrode $(\varnothing 15 \mathrm{~mm})$ were stacked between two $\mathrm{Be}$ spacers to ensure a homogeneous stack pressure. Uneven stack pressure results in nonuniform utilization of the electrodes, as we observed in our development of this experiment (Figure S5). $10 \mu \mathrm{L} \mathrm{mgs}{ }^{-1}$ electrolyte (1 M LiTFSI and $0.25 \mathrm{M} \mathrm{LiNO}_{3}$ in DME:DOL $(1: 1, v: v))$ were added to the cell by an automatic micropipette immediately before the coin cell was closed by a crimper. The coin cell was then sealed in a pouch bag with PE film windows dimensioned to fit the cell holder of the diffractometer and further seals the cell from moisture and air.

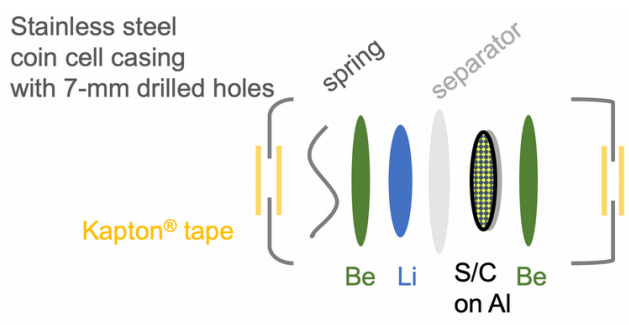

Figure 1: Scheme of the modified coin cell for operando XRD

XRD measurements were carried out in transmission setup on a STOE STADI P diffractometer in transmission mode equipped with a Ge monochromator providing single wavelength $\mathrm{Cu} \mathrm{K}_{\alpha 1}$ radiation $(45 \mathrm{kV}, 40 \mathrm{~mA})$. A Dectris Mythen $1 \mathrm{~K}$ strip detector with a stationary angular $2 \theta$-range of $18.87^{\circ}$ and angular resolution of $0.015^{\circ} 2 \theta$ was used. For the operando experiment, individual XRD patterns were collected for 60 minutes, with the detector in stationary mode centered at $30^{\circ}$ $(2 \theta)$. For other ex situ measurements, the data collection strategy is mentioned with the data. The diffraction data were analyzed using Topas software. ${ }^{29}$ All Pawley refinements were carried out using a six degree Chebyshev polynomial background function and the modified Thompson Cox Hastings pseudo Voigt peak shape function. Peak asymmetry due to the axial diver-gence was used only for the refinement of the diffraction data of a-S in capillary. More details of the aforemen-tioned refinement functions can be found in the Topas manual.The integration of the peak area of 111 reflection of $\mathrm{Li}_{2} \mathrm{~S}$ was carried out by Origin software from $25.5^{\circ}$ to $28^{\circ}$ using the pattern before appearance of the peak as the background.

\subsection{Intermittent Current Interruption (ICI) method}

The electrochemical tests were conducted using a SP-240 potentiostat (Bio-Logic). The cell was rested for six hours after assembly to ensure complete wetting of the electrode and electrolyte, and stabilization of the Li electrode. The cell was first discharged at $C / 50$ $\left(1 \mathrm{C}=1672 \mathrm{~mA} \mathrm{gs}^{-1}\right)$ and then charged at $\mathrm{C} / 25$, and then galvanostatically cycled at $\mathrm{C} / 10$ for the remainder of the test. A one-second current interruption was made every five minutes, during which the potential response of the cell was recorded at $0.1 \mathrm{sec}$ intervals. The potential-response of the cell was analyzed by the $\mathrm{ICl}$ method. ${ }^{18}$ For each current pause, a 
linear regression of the change of potential $(\Delta \mathrm{E})$ against the square root of step time $\left(t^{0.5}\right)$ was carried out automatically with a program written in-house, in the $\mathrm{R}$ programming language. ${ }^{30}$ The linear relationship between $\Delta \mathrm{E}$ and $t^{0.5}$, which has been previously found empirically, is mathematically validated to be the expected potential response of a porous electrode to a current interruption; the derivation of this is given in the Supporting Information. The internal resistance $(R)$ was obtained by dividing the intercept $(\Delta \mathrm{E}(\mathrm{t}=0))$ by the current density, since the resistance values in this work are normalized by the area of the electrode in contact with the separator to allow comparison with data in literature. The diffusion resistance coefficient (k) was derived by dividing the slope by the current density, which is proportional to the equivalent coefficient of the well-established Warburg impedance model. The conventional coin cells, which served as electrochemical control experiments, were cycled under the same protocol with an Arbin BT-2043 potentiostat.

\subsection{Synchronization of XRD and ICI data}

The time coordinate of an operando XRD pattern is assigned to be the midpoint of its 60minute collection time. For example, the pattern collected in the first hour is assigned a time coordinate $\mathrm{t}=0.5 \mathrm{~h}$. The time coordinate of an ICl measurement is assigned as the time when the current interruption begins. In Figure 4 , where the diffusion resistance coefficient is plotted against the integrated area of the 111 reflection of $\mathrm{Li}_{2} \mathrm{~S}$, the diffusion resistance coefficient is obtained by interpolating from the two nearest data points at the time coordinate of the XRD pattern. Both electrochemical and $X R D$ raw data and the $R$ scripts used for the $\mathrm{ICl}$ analysis are available online via Zenodo. ${ }^{31}$

\section{Results and discussion}

\subsection{Theoretical and experimental verification of the ICI method}

The internal resistance measurement of the $\mathrm{ICl}$ method has been demonstrated and verified by EIS in the previous work. ${ }^{18}$ Here, the method is further developed for the measurement of the coefficient of diffusion resistance (hereafter referred to as $\mathrm{k}$ ), which can be defined as

$$
k=\frac{-1}{j} \cdot \frac{d \Delta E}{d t^{0.5}}
$$

where $\mathrm{j}$ is the current density applied on the porous electrode before the current pause (during which $\mathrm{j}=0$ ), $\Delta \mathrm{E}$ and $\mathrm{t}$ are the potential change and time, respectively, since the cell is switched to open circuit. As derived in the Supporting Information, k, which has the unit of
$\Omega \mathrm{s}^{-0.5} \mathrm{~cm}^{2}$, is linearly proportional to the coefficient of the Warburg impedance $(\sigma)$, which is the most common model for a diffusioncontrolled response in an EIS experiment.

$$
k=\sqrt{\frac{8}{\pi}} \sigma
$$

$$
\text { (2) }
$$

By reanalyzing previously reported experimental data, ${ }^{27}$ where EIS and the $\mathrm{ICl}$ method was applied to a Li-S cell in the same experiment, $\mathrm{k}$ measured by $\mathrm{ICl}$ is seen to be equivalent to $\sqrt{\frac{8}{\pi}} \sigma$ as measured by EIS over the full duration of the experiment, as depicted in Figure S2.

\subsection{Operando XRD data and concurrent resistance measurement of the first cycle}

An overview of the results of the combined operando XRD and $\mathrm{ICl}$ measurements is displayed in Figure 2. With the XRD patterns presented as a heat map at the top, the potential (E), internal resistance (R) and diffusion resistance coefficient $(\mathrm{k})$ are plotted in curves with the colors corresponding to the sulfur species detected by XRD at the time of measurement. During the $\mathrm{C} / 50$ discharge in the first cycle, the $a-S$ phase disappeared as the upper discharge plateau in the potential profile ended. The value of $R$, which is a sum of electronic, ionic and charge transfer resistances, ${ }^{18}$ dropped to a local minimum in the middle of the first discharge upper plateau and then increased again to a local maximum. This peak in $\mathrm{R}$ between the two potential plateaus was ascribed to the decreased solution conductivity due to the high polysulfide concentration at this SoC, ${ }^{18,24,32}$ which is corroborated by the observed absence of crystalline sulfur species in the XRD data. After one third of the lower discharge plateau, the broad reflections from the $\mathrm{Li}_{2} \mathrm{~S}$ phase appeared in the XRD patterns, which were a result of small grain size and/or low crystallinity of $\mathrm{Li}_{2} \mathrm{~S} .{ }^{13,14,16}$ The observed $\mathrm{SoC}$ at which the $\mathrm{Li}_{2} \mathrm{~S}$ becomes detectable by XRD varies in previous works ${ }^{9,11-14,16}$ and also differs between the first and the subsequent cycles in this work, which is readily explained by the change in kinetics due to the increased current density. Upon the subsequent $\mathrm{C} / 25$ charging, both $\mathrm{R}$ and $\mathrm{k}$ dropped immediately as the intensity of the peaks from $\mathrm{Li}_{2} \mathrm{~S}$ slowly decreased. This difference between discharging and charging of both of these quantities can also be observed in the following cycles, where the cell was charged and discharged at the same C-rate $(C / 10)$. The lower value of $R$ upon charging has been reported previously by various 
techniques, ${ }^{18,33,34}$ and a lower diffusion resistance on charging compared to discharging has also been measured by EIS. ${ }^{34}$ At the upper charging plateau $(\sim 2.35 \mathrm{~V})$ and subsequent cycles, elemental sulfur was detected by XRD in the monoclinic $(\beta)$ form, as observed in previous works. ${ }^{14,16,35}$ Pawley refinement ${ }^{36}$ of the XRD data obtained at the end of the first discharge and charge steps was carried out to ensure phase purity, as shown in Figure S6 and S7, respectively. The formation of $\beta$-S rather than the thermodynamically favored $\alpha-S$ has been argued to be a consequence of kinetics, ${ }^{16}$ which was also observed in quenched samples. ${ }^{37}$

\subsection{Operando XRD data and concurrent resistance measurement after the first cycle}

The applied current was increased to $C / 10$ at the beginning of the second discharge. The correlation between the increase in $\mathrm{k}$ and the formation of $\mathrm{Li}_{2} \mathrm{~S}$ can be seen in Fig. 2. In the second and third cycles, this increase was observed before the detection of $\mathrm{Li}_{2} \mathrm{~S}$, and then observed only after the detection of $\mathrm{Li}_{2} \mathrm{~S}$ from the fourth discharge. This change in the onset of $k$ increase may suggest some form of relaxation process of the porous carbon matrix over the first few cycles. Since the fourth discharge, the variation of $k$ stabilized and showed a repeating pattern: first increasing to a plateau as $\mathrm{Li}_{2} \mathrm{~S}$ started to precipitate, and then a further sharp increase at the end of discharge. At the start of charge, $k$ dropped immediately and remained close to the minimum value until the appearance of the $\beta-S$ phase. At the end of charging, $k$ again increased rapidly and reduced similarly quickly on the subsequent discharge.

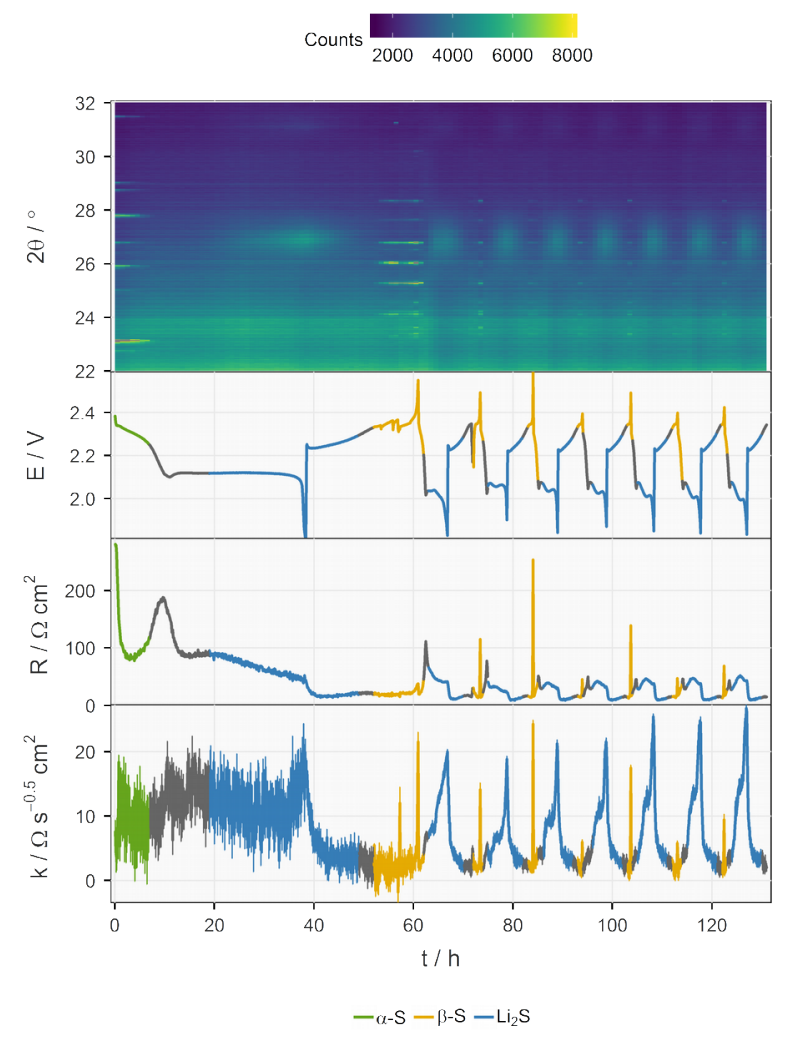

Figure 2: Operando XRD data plotted against time. The XRD patterns are displayed as a heat map at the top, followed by the potential $(E)$, internal resistance (R) and diffusion resistance coefficient (k) plotted in colors corresponding to the crystalline sulfur species detected by XRD. The gray segments indicate that no crystalline sulfur species were detected. Error bars for R and $\mathrm{k}$ are estimated by the standard deviation from linear regression, which are most prominent in $\mathrm{k}$ in the first cycle due to the low current and hence relatively small voltage drop.

As previously discussed for the first cycle, the cell showed a local maximum in $\mathrm{R}$ when no crystalline species were visible in XRD pattern. From the second cycle, $\mathrm{R}$ also showed a sudden increase at the end of charging (e.g. at $t=85$ h) and a broad "bump" at the lower discharge plateau (e.g. at $t=87 \mathrm{~h}$ ). We typically do not observe these features in cells with lower Sloadings, i.e. $<3 \mathrm{mAh} \mathrm{cm}^{-2} .{ }^{18}$ Since these two features coincide with the precipitation of $\beta-S$ and $\mathrm{Li}_{2} \mathrm{~S}$, respectively, these observations suggest that the precipitation behavior of the sulfur species is highly dependent on loading, which may in turn be due to inhomogeneous current distribution along the thickness of the electrode. This finding coincides with higher overpotential and lower discharge capacity in Li-S cells with higher S-loadings. ${ }^{28,38}$

We observe also that both the XRD and electrochemical results after the third cycle are 
relatively unchanging from one cycle to the next, compared to those from the initial three cycles. Indeed, it is usually observed in the cycling data of Li-S cells that the discharge capacity stabilizes after the third cycle, ${ }^{39}$ which is also the case for all of the cells presented in Fig. S5. From a practical point of view, the stabilized properties of the cell are more relevant to the understanding and development of the Li-S system, although most operando works in the literature have so far mainly considered the initial cycle(s). To the best of our knowledge, there is only one previous operando XRD work on Li-S cells for more than five cycles, where differences in the voltage profile and SoC ranges where crystalline phases form can be clearly observed. ${ }^{11}$

\subsection{Identification of the resistance sources by a three-electrode cell}

It is crucial for the correct interpretation of the above results to distinguish the resistance contributions from the positive and negative electrodes. Although the operando cell is by conventional definition a half cell, i.e. the negative electrode is excess in capacity, $\mathrm{Li}$ metal does not always behave as an ideal counter electrode. ${ }^{40}$ Therefore, a threeelectrode cell was made in a comparable cell format and the $\mathrm{ICl}$ measurements were performed, which are presented in Figure S8. The inclusion of the third reference electrode allows the separate measurement of the positive and negative electrode potentials, and their associated resistances. From the results, we observe that $R$ was mostly equally distributed between the positive and negative electrodes, which is in accordance with the previous conclusions that the major changes in $\mathrm{R}$ during cycling are related to changes in the ionic resistance. ${ }^{18}$ The S/C composite electrode, on the other hand, contributed the majority $(80-90 \%)$ of the overall $k$ in this cell. This therefore confirms that the major changes of $k$ in the operando cell originated from the positive electrode, and characterizes the transport properties in the porous carbon matrix, as discussed in the introduction.

\subsection{Correlation between the evolution of $\mathrm{Li}_{2} \mathrm{~S}$ and $\mathrm{k}$}

To investigate the correlation between the evolution of $\mathrm{Li}_{2} \mathrm{~S}$ and the transport properties of the positive electrode, the evolution of the integrated peak area of the 111 reflection of $\mathrm{Li}_{2} \mathrm{~S}$ and $\mathrm{k}$ were plotted together in Figure 3 . Although the maximum of the peak area was not captured for all cycles due to the limited time resolution of the in-house measurement, the integrated intensity of the 111 reflection increased and decreased approximately linearly with time-and thus SoC-except for the first two cycles. Previous operando XRD studies have however reported that the precipitation and dissolution rates varies with $\mathrm{SoC}_{1}^{8,9,12}$ though these discrepancies may simply be due to different experimental conditions, e.g. the different carbon matrices, electrode preparation processes and C-rates. ${ }^{12}$ Two previous studies also concluded that the lower precipitation rate of $\mathrm{Li}_{2} \mathrm{~S}$ at the end of discharge was a consequence of insulating $\mathrm{Li}_{2} \mathrm{~S}$ covering the conductive surface of the carbon matrix..$^{8,9}$ However, the porous carbon in both of these studies was Super $P$, which has a much lower specific surface area than the Ketjenblack carbon matrix used in this work. ${ }^{41}$ In this context, the specific surface area of the carbon matrix plays a critical role in the specific capacity of a Li-S cell. ${ }^{42}$ Since the specific capacity is a direct outcome of sulfur utilization, it is expected that the reaction mechanisms can vary between cells comprising these two carbon matrices.
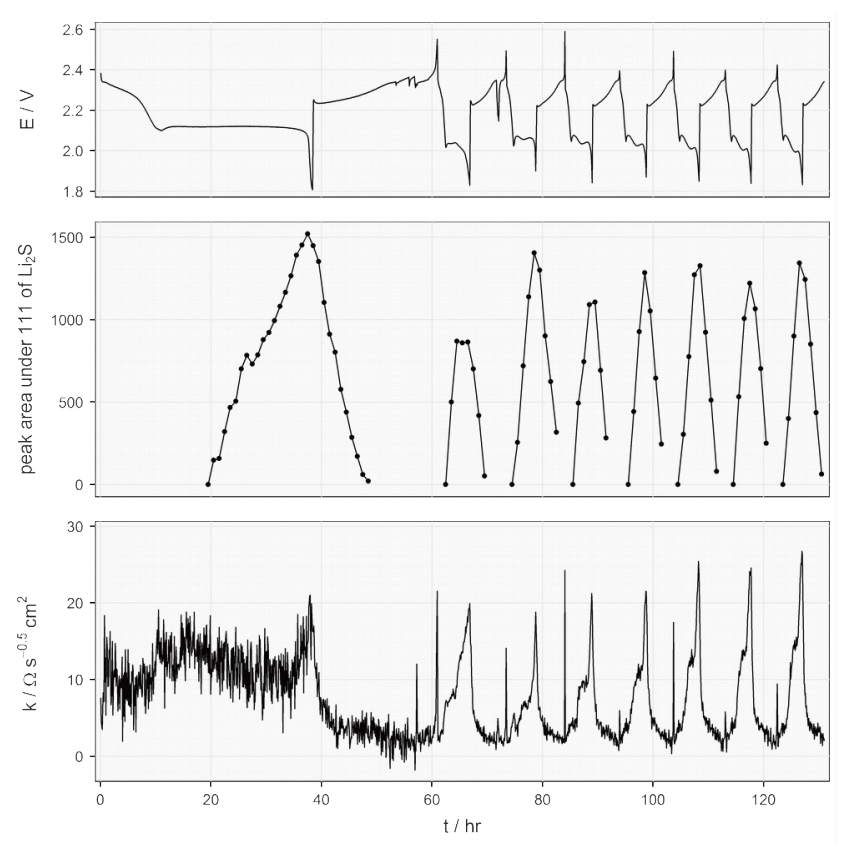

Figure 3: Potential $(E)$, integrated area of the 111 reflection of $\mathrm{Li}_{2} \mathrm{~S}$ and the diffusion resistance coefficient (k) plotted against time.

To directly compare the changes in quantity of $\mathrm{Li}_{2} \mathrm{~S}$ and the changes in diffusion resistance, $\mathrm{k}$ is plotted against the integrated area of the 111 reflection of $\mathrm{Li}_{2} \mathrm{~S}$ in Figure 4. At the bottom left corner of the plot, $\mathrm{k}$ had a relatively constant value below $5 \Omega \mathrm{s}^{-0.5} \mathrm{~cm}^{2}$ when there was no $\mathrm{Li}_{2} \mathrm{~S}$ detected by the XRD. Upon discharging, $\mathrm{Li}_{2} \mathrm{~S}$ was initially detected with almost no change in $\mathrm{k}$. An obvious increase in $\mathrm{k}$ was observed after approximately $20 \%$ of the maximum amount of $\mathrm{Li}_{2} \mathrm{~S}$ was detected. It first increased to a stable value of $10-15 \Omega \mathrm{s}^{-0.5} \mathrm{~cm}^{2}$, followed by a steep increase at the end of 
discharge, concomitant with the maximum in the amount of $\mathrm{Li}_{2} \mathrm{~S}$. On charging, $k$ dropped immediately below $5 \Omega \mathrm{s}^{-0.5} \mathrm{~cm}^{2}$, which is the value of $k$ measured before the XRD signal of $\mathrm{Li}_{2} \mathrm{~S}$ appeared upon discharge, even though there was still a considerable amount of detected $\mathrm{Li}_{2} \mathrm{~S}$. This asymmetric evolution of $\mathrm{k}$ indicates that the transport properties of the porous carbon matrix vary substantially when a Li-S cell with a high S-loading electrode is discharged and charged.

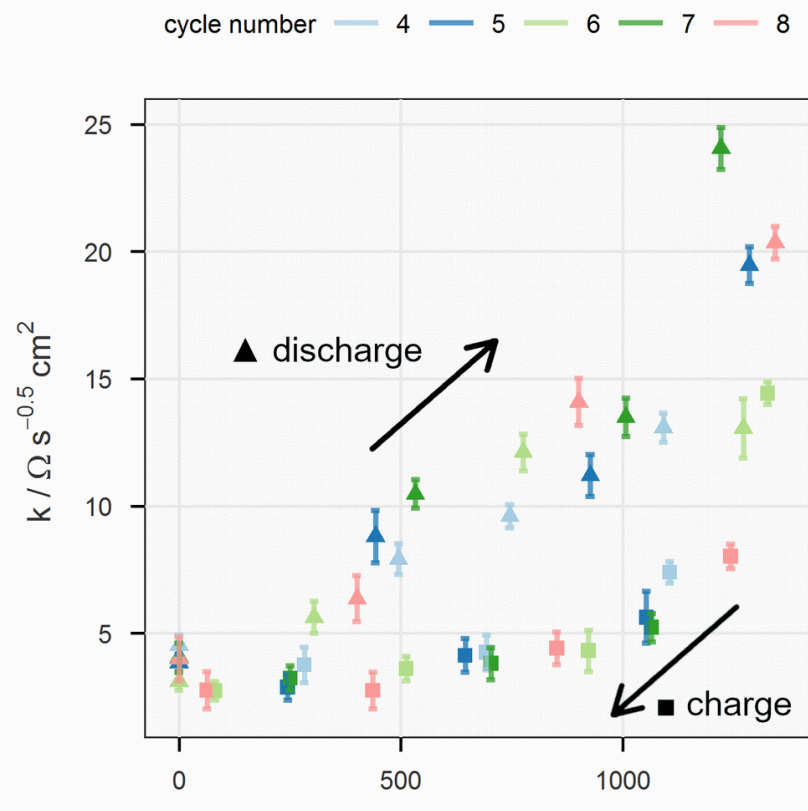

integrated area under 111 peak of $\mathrm{Li}_{2} \mathrm{~S} /$ a.u.

Figure 4: The diffusion resistance coefficient $(k)$ plotted against the integrated area of the 111 reflection of $\mathrm{Li}_{2} \mathrm{~S}$ with data from the fourth to the eighth cycles.

\subsection{Effect of the precipitation and dissolution of $\mathrm{Li}_{2} \mathrm{~S}$ on the carbon matrix}

The growth kinetics of $\mathrm{Li}_{2} \mathrm{~S}$ from ether-based electrolytes have previously been studied for open-structure carbon matrices, namely carbon nanotube scaffolds, by scanning electron microscopy (SEM). ${ }^{43,44}$ Although the geometric differences should be taken into account, both of these studies argued that, following nucleation, $\mathrm{Li}_{2} \mathrm{~S}$ growth proceeds twodimensionally on the surface of the conductive carbon matrix, ${ }^{43}$ at least in the initial stage. ${ }^{44}$ The XRD patterns obtained in this work show that $\mathrm{Li}_{2} \mathrm{~S}$ did not form large crystallites, so it is reasonable to consider a similar growth mechanism here. We also consider this mechanism in the context of a simple numerical simulation based on the transmission line model for a porous electrode material. A description of the model is presented in the Supporting Information.

In this case, we can interpret the initial slow increase of $k$ with respect to the evolution of $\mathrm{Li}_{2} \mathrm{~S}$ as due to the progressive coverage of $\mathrm{Li}_{2} \mathrm{~S}$ within the porous carbon matrix, as depicted schematically in Figure 5 . In this stage, $k$ is increased mostly by the reduction of capacitance of the electrochemical double-layer inside the pore, depicted as C in Figure 5, following equation S15 in the Supporting Information. We assume then that the surface coverage of $\mathrm{Li}_{2} \mathrm{~S}$ at the pore surface reaches a maximum, and correspondingly a minimum in $C$, relatively early in the discharge. In the next stage, the continued precipitation of $\mathrm{Li}_{2} \mathrm{~S}$ inside the pores reduces the ionic resistance inside the pores, through either narrowing or otherwise partial filling of the pores. In our numerical model, the ionic conductivity is assumed to reduce linearly with the fraction of $\mathrm{Li}_{2} \mathrm{~S}$ in the pore. The pore resistance $\mathrm{R}^{\prime}$ (to distinguish this from the internal resistance $R$ already discussed in this work) then increases exponentially as discharge proceeds. This also, in turn, increases $k$, following this model. At the limit of discharge, we can expect that $\mathrm{Li}_{2} \mathrm{~S}$ deposition should ultimately fill pores, according to the numerical model. However, we can also expect that in the real case, pores may be blocked rather than completely filled, or channels between pores may be blocked. Any of these cases will result in a fast deterioration of the transport properties of the carbon matrix, leading to the termination of discharge.

This model is firstly supported by various operando spectroscopic experiments, which have shown that polysulfides remain in the positive electrode at the end of discharge. ${ }^{16,45-47}$ Secondly, a combination of modeling and experimental work also concluded that the discharge capacity is limited by mass transport, rather than charge transfer. ${ }^{48}$ Furthermore, it has been reported that the total amount of $\mathrm{Li}_{2} \mathrm{~S}$ which can be generated in the electrode during discharge can be increased by promoting its heterogeneous nucleation through surface modification of the carbon host, which results in more evenly distributed precipitates. ${ }^{12}$ This finding corroborates our conclusion that the discharge process is terminated due to the pore-blocking $\mathrm{Li}_{2} \mathrm{~S}$ precipitates, which indicates that the capacity limiting factor is pore blockage rather than a deficiency of active materials at the electrode level.

The instant drop in $k$ upon charging is consistent with the clearance of the blockage of the channels inside the carbon matrix, beginning with the oxidation of $\mathrm{Li}_{2} \mathrm{~S}$ at the surface of the pores. As polysulfides remain present in the electrode in the fully discharged state as discussed above, the oxidation of $\mathrm{Li}_{2} \mathrm{~S}$ 
can proceed easily through redox mediation. ${ }^{46}$ Moreover, the oxidation of $\mathrm{Li}_{2} \mathrm{~S}$, which is driven by the applied current, can be expected to begin with those precipitates at the electrolyte/carbon host interface. As the surface coverage of $\mathrm{Li}_{2} \mathrm{~S}$ reduces, the capacitance of the electrochemical doublelayer, C, at the surface should quickly reach its original value before $\mathrm{Li}_{2} \mathrm{~S}$ precipitates, even while $\mathrm{Li}_{2} \mathrm{~S}$ particles may remain in the pore, as shown in the scheme in Fig. 5. In our numerical model, this relatively fast increase in $\mathrm{C}$ back to the initial value is manifested in $k$ as a similarly fast drop, to a lower value than at the equivalent state of discharge during the discharge process.

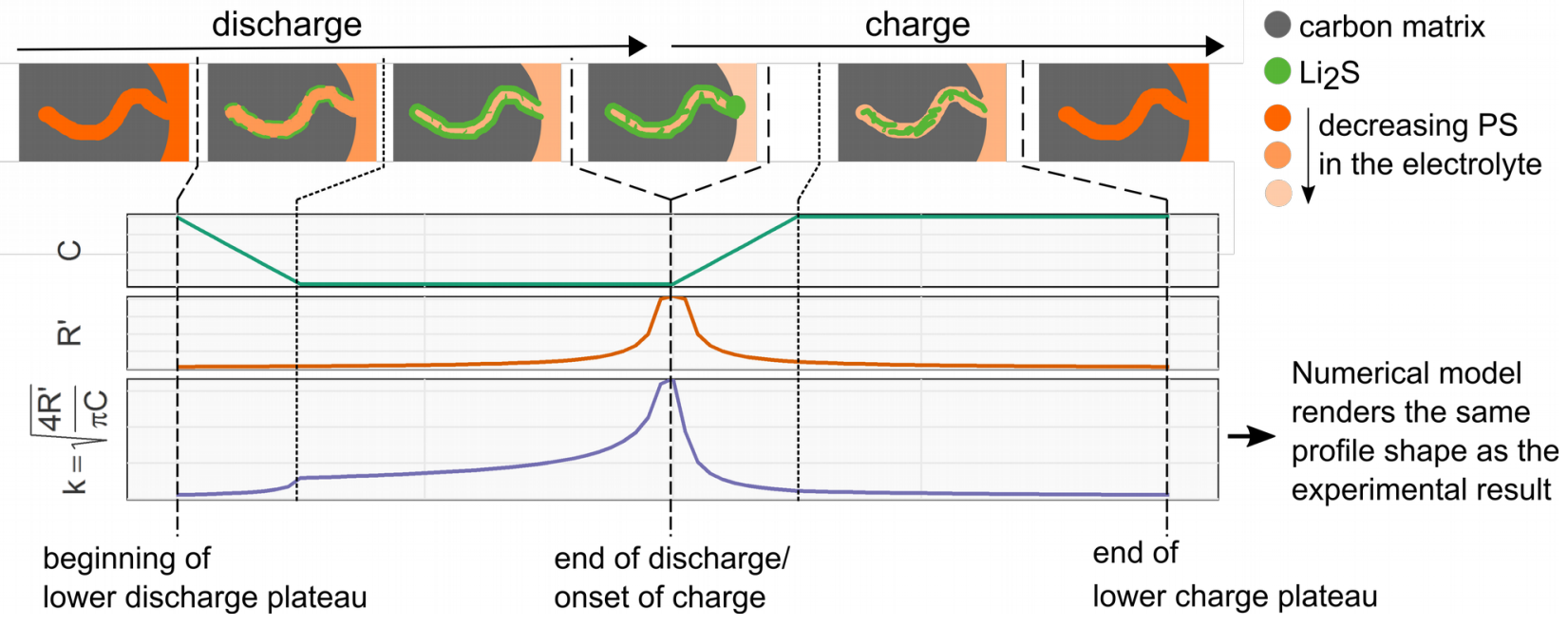

Figure 5: Schematic illustration of the proposed mechanism of precipitation and dissolution of $\mathrm{Li}_{2} \mathrm{~S}$ inside pores of the carbon matrix. Based on the mechanism, a simple numerical model with the capacitance (C) and ionic resistance ( $\left.R^{\prime}\right)$ inside the pores renders the same profile of the diffusion resistance coefficient (k) as the one obtained from the experiment. The equation of $k$ is equation S15 in the Supporting Information. A detailed description of the model can be found in the Supporting Information.

Although the parameters $\mathrm{R}^{\prime}$ and $\mathrm{C}$ are not directly measurable in this experiment, all of the features of the change in $k$ observed experimentally in Figure 4 are reproduced by the numerical model, and consistent with the above interpretation for the precipitation and dissolution mechanisms. On this basis, therefore, the combination of operando XRD and $\mathrm{ICl}$ constitutes a valuable method not only for quantifying charge and discharge products in the sulfur electrode, but gaining a deeper understanding of the precipitation and dissolution processes in the host structure itself and their effect on electrochemical performance.

\section{Conclusion}

In this study, an operando XRD experiment of a Li-S cell with simultaneous resistance determination has been reported for the first time. In this experiment, the formation and disappearance of crystalline sulfur species was correlated directly to changes in internal resistance and a coefficient of diffusion resistance. By investigating the relationship between the evolution of $\mathrm{Li}_{2} \mathrm{~S}$ and diffusion resistance coefficient, which quantifies mass transport inside the porous carbon matrix of the positive electrode, we conclude that the discharge process was terminated by pore blockage in the porous carbon matrix, which limits the utilization of sulfur and correspondingly the specific capacity of the sulfur/carbon composite electrode. Since it is well-established in the field that the structure of the conductive host is crucial for sulfur utilization, this new method provides vital information which will aid in both the understanding and development of the positive electrode of Li-S batteries.

In addition, this work also demonstrated the value of an operando cell constructions which strive for a balance between signal-to-noise ratio and electrochemical equivalence. The electrochemical equivalence not only ensures that the observations from the operando technique are representative, but also demonstrates the value of the $\mathrm{ICl}$ method in providing accurate and "real time" information about energy loss processes in a battery, which can be compared across very different experiments. Furthermore, given that the Warburg impedance and transmission line models can describe other transport processes, such as ion transport in intercalation materials, this method can be readily applied to other battery chemistries such as lithium-ion batteries. With these advantages and the compatibility with various operando techniques, the $\mathrm{ICl}$ method has considerable 
value for characterizing not just the Li-S system but also a wide variety of other energy storage chemistries.

\section{ASSOCIATED CONTENT}

\section{Supporting Information.}

Derivation of the relationship of $k$ to common equivalent cir-

cuit models, confirmation of the electrochemical equivalence

of the operando cell, Pawley refinement of the XRD pattern at

the end of the first discharge and charge, $\mathrm{ICl}$ results of a three-

electrode cell, numerical model for the evolution of $k$. This material is available free of charge via the Internet at http://pubs.acs.org."

\section{Electronic Supporting Data.}

Raw data from electrochemical experiments, including XRD patterns and the scripts for $\mathrm{ICl}$ analysis and associated plots are available free of charge at DOI: 10.5281/zenodo.3514967

\section{AUTHOR INFORMATION}

\section{Corresponding Author}

* matthew.lacey@scania.com

\section{Author Contributions}

The manuscript was written through contributions of all authors.

\section{ACKNOWLEDGMENT}

The authors are grateful to Swedish Energy Agency for the financial support through STARC (42762-1) and STEM Li-S (42031-1) projects and the Swedish Foundation for Strategic Research (SSF) for the financial support through the Swedish national graduate school in neutron scattering (SwedNess). The strategic research area StandUp for Energy is also acknowledged.

\section{REFERENCES}

(1) Wujcik, K. H.; Wang, D. R.; Teran, A. A.; Nasybulin, E.; Pascal, T. A.; Prendergast, D.; Balsara, N. P. Determination of Redox Reaction Mechanisms in Lithium-Sulfur Batteries. In Electrochemical Engineering; John Wiley \& Sons, Ltd, 2018; pp 41-74.

(2) Nitta, N.; Wu, F.; Lee, J. T.; Yushin, G. Li-lon Battery Materials: Present and Future. Mater. Today 2015, 18 (5), 252-264.

(3) Berg, E. J.; Trabesinger, S. Viability of PolysulfideRetaining Barriers in Li-S Battery. J. Electrochem. Soc. 2018, 165 (1), A5001-A5005.

(4) Pope, M. A.; Aksay, I. A. Structural Design of Cathodes for Li-S Batteries. Adv. Energy Mater. 2015, 5 (16), 1-22.

(5) Manthiram, A.; Chung, S.-H.; Zu, C. LithiumSulfur Batteries: Progress and Prospects. Adv. Mater. 2015, 27 (12), 1980-2006.

(6) Wild, M.; O'Neill, L.; Zhang, T.; Purkayastha, R.; Minton, G.; Marinescu, M.; Offer, G. J. Lithium Sulfur Batteries, a Mechanistic Review. Energy Environ. Sci. 2015, 8 (12), 3477-3494.

(7) Nelson, J.; Misra, S.; Yang, Y.; Jackson, A.; Liu, Y.; Wang, H.; Dai, H.; Andrews, J. C.; Cui, Y.; Toney,
M. F. In Operando X-Ray Diffraction and Transmission X-Ray Microscopy of Lithium Sulfur Batteries. J. Am. Chem. Soc. 2012, 134 (14), 6337-6343.

Walus, S.; Barchasz, C.; Bouchet, R.; Leprêtre, J. C.; Colin, J. F.; Martin, J. F.; Elkaïm, E.; Baehtz, C.; Alloin, F. Lithium/Sulfur Batteries Upon Cycling: Structural Modifications and Species Quantification by in Situ and Operando X-Ray Diffraction Spectroscopy. Adv. Energy Mater. 2015, 5 (16), 1-5.

(9) Yu, S. H.; Huang, X.; Schwarz, K.; Huang, R.; Arias, T. A.; Brock, J. D.; Abruña, H. D. Direct Visualization of Sulfur Cathodes: New Insights into Li-S Batteries via Operando X-Ray Based Methods. Energy Environ. Sci. 2018, 11 (1), 202-210.

(10) Kulisch, J.; Sommer, H.; Brezesinski, T.; Janek, J. Simple Cathode Design for Li-S Batteries: Cell Performance and Mechanistic Insights by in Operando X-Ray Diffraction. Phys. Chem. Chem. Phys. 2014, 16 (35), 18765-18771.

(11) Conder, J.; Bouchet, R.; Trabesinger, S.; Marino, C.; Gubler, L.; Villevieille, C. Direct Observation of Lithium Polysulfides in Lithium-Sulfur Batteries Using Operando X-Ray Diffraction. Nat. Energy 2017, 2 (6), 17069.

(12) Dörfler, S.; Strubel, P.; Jaumann, T.; Troschke, E.; Hippauf, F.; Kensy, C.; Schökel, A.; Althues, H.; Giebeler, L.; Oswald, S.; et al. On the Mechanistic Role of Nitrogen-Doped Carbon Cathodes in Lithium-Sulfur Batteries with Low Electrolyte Weight Portion. Nano Energy 2018, 54, 116-128.

(13) Cañas, N. A.; Wolf, S.; Wagner, N.; Friedrich, K. A. In-Situ X-Ray Diffraction Studies of Lithium-Sulfur Batteries. J. Power Sources 2013, 226, 313-319.

(14) Waluś, S.; Barchasz, C.; Colin, J.-F.; Martin, J.-F.; Elkaïm, E.; Leprêtre, J.-C.; Alloin, F. New Insight into the Working Mechanism of Lithium-Sulfur Batteries: In Situ and Operando X-Ray Diffraction Characterization. Chem. Commun. 2013, 49 (72), 7899.

(15) Lowe, M. A.; Gao, J.; Abruña, H. D. Mechanistic Insights into Operational Lithium-Sulfur Batteries by in Situ X-Ray Diffraction and Absorption Spectroscopy. RSC Adv. 2014, 4 (35), 18347.

(16) Zhu, W.; Paolella, A.; Kim, C.-S.; Liu, D.; Feng, Z.; Gagnon, C.; Trottier, J.; Vijh, A.; Guerfi, A.; Mauger, A.; et al. Investigation of the Reaction Mechanism of Lithium Sulfur Batteries in Different Electrolyte Systems by in Situ Raman Spectroscopy and in Situ X-Ray Diffraction. Sustain. Energy Fuels 2017, 1 (4), 737-747.

(17) Eroglu, D.; Zavadil, K. R.; Gallagher, K. G. Critical Link between Materials Chemistry and Cell-Level Design for High Energy Density and Low Cost Lithium-Sulfur Transportation Battery. J. Electrochem. Soc. 2015, 162 (6), A982-A990.

(18) Lacey, M. J. Influence of the Electrolyte on the Internal Resistance of Lithium-Sulfur Batteries Studied with an Intermittent Current Interruption Method. ChemElectroChem 2017, 4 (8), 19972004.

(19) Kolosnitsyn, V. S.; Karaseva, E. V.; Kuzmina, E. V.; Ivanov, A. L. Reasons for the Effect of the Amount of Electrolyte on the Performance of Lithium-Sulfur Cells. Russ. J. Electrochem. 2016, 52 (3), 273-282.

(20) Borkiewicz, O. J.; Wiaderek, K. M.; Chupas, P. J.; Chapman, K. W. Best Practices for Operando Battery Experiments: Influences of X-Ray Experiment Design on Observed Electrochemical Reactivity. J. Phys. Chem. Lett. 2015, 6 (11), 
(21) Bleda-Martínez, M. J.; Lozano-Castelló, D.; Morallón, E.; Cazorla-Amorós, D.; Linares-Solano, A. Chemical and Electrochemical Characterization of Porous Carbon Materials. Carbon N. Y. 2006, 44 (13), 2642-2651.

(22) Raccichini, R.; Furness, L.; Dibden, J. W.; Owen, J. R.; García-Araez, N. Impedance Characterization of the Transport Properties of Electrolytes Contained within Porous Electrodes and Separators Useful for Li-S Batteries. J. Electrochem. Soc. 2018, 165 (11), A2741A2749.

(23) de Levie, R. On Porous Electrodes in Electrolyte Solutions. Electrochim. Acta 1963, 8 (10), 751780.

(24) Kolosnitsyn, V. S.; Kuzmina, E. V.; Karaseva, E. V.; Mochalov, S. E. Impedance Spectroscopy Studies of Changes in the Properties of Lithium-Sulfur Cells in the Course of Cycling. Russ. J. Electrochem. 2011, 47 (7), 793-798.

(25) Deng, Z.; Zhang, Z.; Lai, Y.; Liu, J.; Li, J.; Liu, Y. Electrochemical Impedance Spectroscopy Study of a Lithium/Sulfur Battery: Modeling and Analysis of Capacity Fading. J. Electrochem. Soc. 2013, 160 (4), A553-A558.

(26) Park, S.-M.; Yoo, J.-S. Electrochemical Impedance Spectroscopy for Better Electrochemical Measurements: With Impedance Data, a Complete Description of an Electrochemical System Is Possible. Anal. Chem. 2003, 75 (21), 455A-461A.

(27) Lacey, M. J.; Edström, K.; Brandell, D. Visualising the Problems with Balancing Lithium-Sulfur Batteries by "Mapping" Internal Resistance. Chem. Commun. 2015, 51 (92), 16502-16505.

(28) Lacey, M. J.; Österlund, V.; Bergfelt, A.; Jeschull, F.; Bowden, T.; Brandell, D. A Robust, WaterBased, Functional Binder Framework for HighEnergy Lithium-Sulfur Batteries. ChemSusChem 2017, 10 (13), 2758-2766.

(29) Coelho, A. A. TOPAS and TOPAS-Academic: An Optimization Program Integrating Computer Algebra and Crystallographic Objects Written in C++: An. J. Appl. Crystallogr. 2018, 51 (1), 210218.

(30) R Core Team. R: A Language and Environment for Statistical Computing https://www.rproject.org/.

(31) Chien, Y.-C.; Menon, A. S.; Brant, W. R.; Brandell, D.; Lacey, M. J. Supporting data for "Simultaneous monitoring of crystalline active materials and resistance evolution in lithiumsulfur https://doi.org/10.5281/zenodo.3514967.

(32) Zhang, T.; Marinescu, M.; O'Neill, L.; Wild, M. Offer, G. Modeling the Voltage Loss Mechanisms in Lithium-Sulfur Cells: The Importance of Electrolyte Resistance and Precipitation Kinetics. Phys. Chem. Chem. Phys. 2015, 17 (35), 2258122586.

(33) Cañas, N. A.; Hirose, K.; Pascucci, B.; Wagner, N.; Friedrich, K. A.; Hiesgen, R. Investigations of Lithium-Sulfur Batteries Using Electrochemical Impedance Spectroscopy. Electrochim. Acta 2013, 97, 42-51.

(34) Risse, S.; Cañas, N. A.; Wagner, N.; Härk, E.; Ballauff, M.; Friedrich, K. A. Correlation of Capacity Fading Processes and Electrochemical
Impedance Spectra in Lithium/Sulfur Cells. J. Power Sources 2016, 323, 107-114.

(35) Villevieille, C.; Novák, P. A Metastable $\beta$-Sulfur Phase Stabilized at Room Temperature during Cycling of High Efficiency Carbon Fibre-Sulfur Composites for Li-S Batteries. J. Mater. Chem. A 2013, 1 (42), 13089-13092.

(36) Pawley, G. S. Unit-Cell Refinement from Powder Diffraction Scans. J. Appl. Crystallogr. 1981, 14 (6), 357-361.

(37) David, W. I. F.; Ibberson, R. M.; Cox, S. F. J.; Wood, P. T. Order-Disorder Transition in Monoclinic Sulfur: A Precise Structural Study by HighResolution Neutron Powder Diffraction. Acta Crystallogr. Sect. B Struct. Sci. 2006, 62 (6), 953-959.

(38) Kang, S. H.; Zhao, X.; Manuel, J.; Ahn, H. J.; Kim, K. W.; Cho, K. K.; Ahn, J. H. Effect of Sulfur Loading on Energy Density of Lithium Sulfur Batteries. Phys. Status Solidi Appl. Mater. Sci. 2014, 211 (8), 1895-1899.

(39) Urbonaite, S.; Poux, T.; Novák, P. Progress Towards Commercially Viable Li-S Battery Cells. Adv. Energy Mater. 2015, 5 (16), 1-20.

(40) Cheng, X.-B.; Huang, J.-Q.; Zhang, Q. Review-Li Metal Anode in Working Lithium-Sulfur Batteries. J. Electrochem. Soc. 2018, 165 (1), A6058A6072.

(41) Hayashi, M.; Minowa, H.; Takahashi, M.; Shodai, T. Surface Properties and Electrochemical Performance of Carbon Materials for Air Electrodes of Lithium-Air Batteries. Electrochemistry 2010, 78 (5), 325-328.

(42) Dornbusch, D. A.; Hilton, R.; Gordon, M. J.; Suppes, G. J. Effects of Carbon Surface Area on Performance of Lithium Sulfur Battery Cathodes. J. Ind. Eng. Chem. 2013, 19 (6), 1968-1972.

(43) Fan, F. Y.; Carter, W. C.; Chiang, Y. M. Mechanism and Kinetics of Li2S Precipitation in LithiumSulfur Batteries. Adv. Mater. 2015, 27 (35), 5203-5209.

(44) Kong, L.; Chen, J.-X.; Peng, H.-J.; Huang, J.-Q.; Zhu, W.; Jin, Q.; Li, B.-Q.; Zhang, X.; Zhang, Q. Current-Density Dependence of Li2S/Li2S2 Growth in Lithium-Sulfur Batteries. Energy Environ. Sci. 2019, 27-31.

(45) Saqib, N.; Ohlhausen, G. M.; Porter, J. M. In Operando Infrared Spectroscopy of Lithium Polysulfides Using a Novel SpectroElectrochemical Cell. J. Power Sources 2017, 364, 266-271.

(46) Gorlin, Y.; Patel, M. U. M.; Freiberg, A.; He, Q.; Piana, M.: Tromp, M.: Gasteiger, H. A. Understanding the Charging Mechanism of Lithium-Sulfur Batteries Using Spatially Resolved Operando X-Ray Absorption Spectroscopy. J. Electrochem. Soc. 2016, 163 (6), A930-A939.

(47) Dominko, R.; Vizintin, A.; Aquilanti, G.; Stievano, L.; Helen, M. J.; Munnangi, A. R.; Fichtner, M.; Arcon, I. Polysulfides Formation in Different Electrolytes from the Perspective of X-Ray Absorption Spectroscopy. J. Electrochem. Soc. 2018, 165 (1), A5014-A5019.

(48) Zhang, T.; Marinescu, M.; Walus, S.; Kovacik, P.; Offer, G. J. What Limits the Rate Capability of LiS Batteries during Discharge: Charge Transfer or Mass Transfer? J. Electrochem. Soc. 2018, 165 (1), A6001-A6004. 


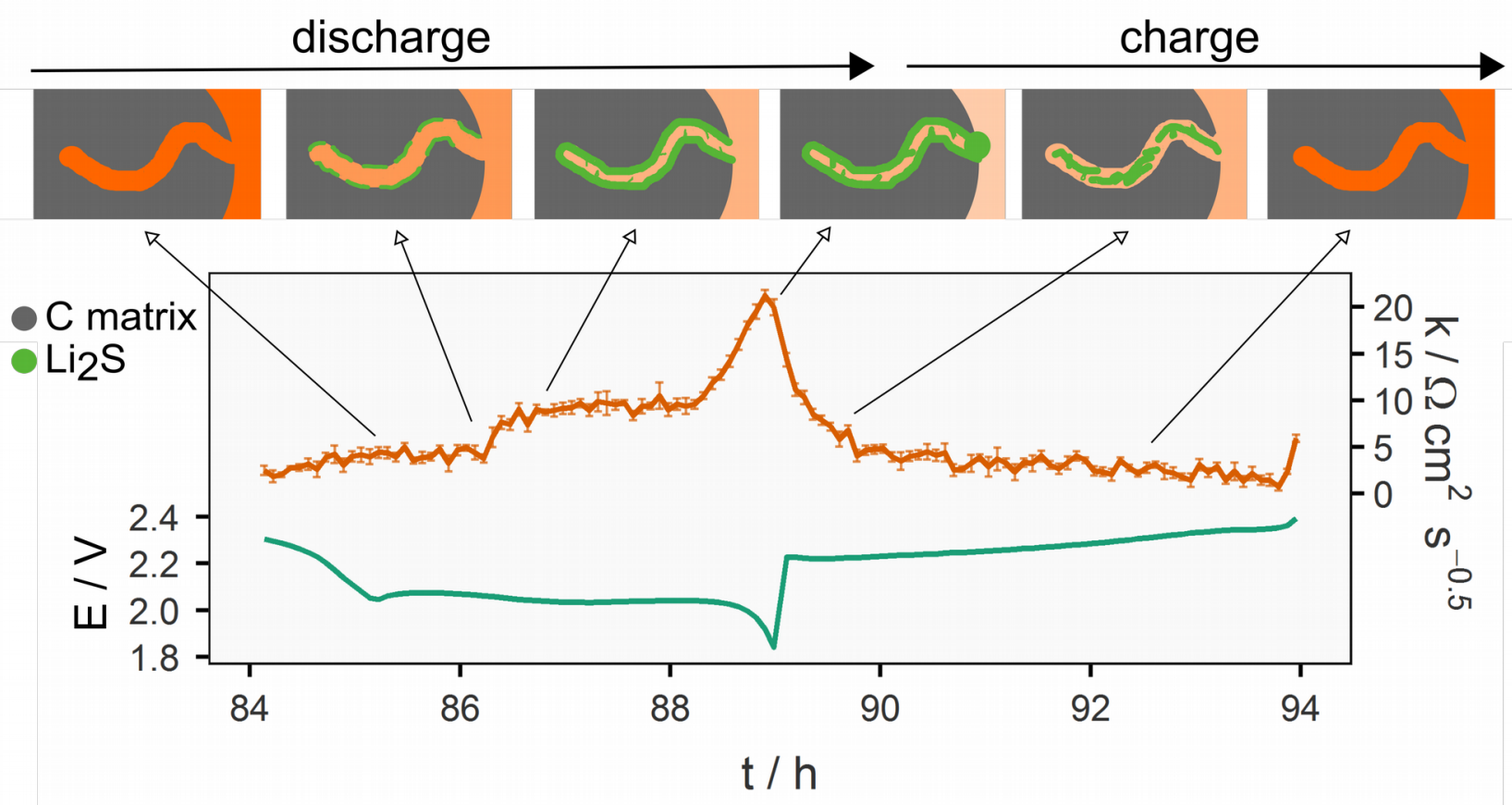

